\title{
STICKY BONE IN DEHISCENCE DEFECT AROUND DENTAL IMPLANT
}

\author{
Waleed M. Atia ${ }^{1} B D S$, Abdel Aziz F. Khalil ${ }^{2} P h D$, Lydia N. Melek ${ }^{3} P h D$
}

\section{ABSTRACT}

INTRODUCTION: Back in time dentists used to place implants in locations with sufficient bone-dimensions only, with less regard to placement of final definitive restoration but most of the times, the placement of implant is not as accurate as intended and even a minor variation in comparison to ideal placement causes difficulties in fabrication of final prosthesis. The use of bone substitutes and membranes is now one of the standard therapeutic approaches. In order to accelerate healing of bone graft over the bony defect, numerous techniques utilizing platelet and fibrinogen concentrates have been introduced in the literature..

OBJECTIVES: This study was designed to evaluate the efficacy of using Autologous Concentrated Growth Factors (CGF) Enriched Bone Graft Matrix (Sticky Bone) and CGF-Enriched Fibrin Membrane in management of dehiscence defect around dental implant in narrow maxillary anterior ridge.

MATERIALS AND METHODS: Eleven DIO implants were inserted in six adult patients presenting an upper alveolar ridge width of less than $4 \mathrm{~mm}$ determined by cone beam computed tomogeraphy (CBCT). After implant placement, the resultant vertical labial dehiscence defect was augmented utilizing Sticky Bone and CGF-Enriched Fibrin Membrane. Three CBCTs were made, pre-operatively, immediately postoperatively and six-months post-operatively. The change in vertical defect size was calculated radiographically then statistically analyzed.

RESULTS: Vertical dehiscence defect was sufficiently recovered in 5 implant-sites while in the other 6 sites it was decreased to mean value of $1.25 \mathrm{~mm} \pm 0.69 \mathrm{SD}$, i.e the defect coverage in 6 implants occurred with mean value of $4.59 \mathrm{~mm} \pm 0.49 \mathrm{SD}$. Also the results of the present study showed that the mean of average implant stability was $59.89 \mathrm{~mm} \pm 3.92$

CONCLUSIONS: The combination of PRF mixed with CGF with bone graft (allograft) can increase the quality (density) of the newly formed bone and enhance the rate of new bone formation.

KEYWORDS: Sticky bone,dental implant, dehiscence, defect

1. B.D.S. Faculty of Dentistry Alexandria University

2. Professor of Oral and Maxillofacial Surgery, Faculty of Dentistry Alexandria University

3. Lecturer of Oral and Maxillofacial Surgery, Faculty of Dentistry Alexandria University

\section{INTRODUCTION}

The development of implant supported oral restoration has become an increasingly used treatment option for partially edentulous and completely edentulous patients. Even in patients with severe bone loss and in locations which were previously considered unsuitable for implant placement where it is now made possible by means of bone augmentation, regeneration and soft tissue regeneration procedures (1). Bone grafting is the process of surgically placing biocompatible graft material or autologous bone graft onto spaces around a broken bone or bone defect(2).

Defects are augmented with bone from the patient's own body (autogenous bone), or an artificial, synthetic or natural alternative. The graft not only augment bone deficiencies, but also helps the mammal organism to regrow its own lost bone (3). In order to accelerate healing of bone graft in the bony defect, numerous techniques utilizing platelet and fibrinogen concentrations have been described in the literature. Platelet is known to contain high quantities of growth factors, such as transforming growth factors $\beta 1$ (TGFs-1), platelet-derived growth factor (PDGF), epithelial growth factor (EGF), insulin growth factor-I (IFG-I) and vascular endothelial growth factors (VEGF), which stimulates cell proliferation and regulates angiogenesis $(4,5)$.

Platelet rich plasma (PRP) and plasma rich in growth factors (PRGF) belong to the first generation of platelet concentrates. PRP and PRGF require chemical additives such as anticoagulants and thrombin or calcium chloride to induce fibrin cross-linking before application to the surgical site (6). Platelet rich fibrin (PRF) and concentrated growth factors (CGF), as second generation of platelet concentrate, utilizes patient's venous blood alone to trigger platelet activation and fibrin cross-linking (7).

PRF and CGF can be used as alternative to traditional barrier membranes to cover bone graft, promising acceleration of tissue regeneration. Unlike PRF, using constant centrifugation speed, CGF is theorized to produce much larger, denser and richer fibrin matrix containing growth factors by altered centrifugation speeds (8).

So, the aim of this study was to evaluate the efficacy of using Autologous Concentrated Growth Factors (CGF) Enriched Bone Graft Matrix (Sticky Bone) and CGFEnriched Fibrin Membrane in the management of dehiscence defects around endosseous dental implants in narrow maxillary anterior ridge to enhance esthetics and function.

\section{MATERIALS AND METHODS}

This case series was conducted on eleven implants placed in six patients of both genders. Appropriate ethical clearance was granted from the institution in which the study was carried out, also an informed consent was obtained from all patients.The patients were selected from the Out Patient Clinic of the Oral and Maxillofacial Surgery Department, Faculty of Dentistry, Alexandria University.

The inclusion criteria of this study were; patients having good oral hygiene and good compliance to the treatment, patients having history of extraction of maxillary anterior teeth of at least six months, Ridge width less than 4 millimeter ( $\mathrm{mm}$ ) and vertical dehiscence defect should be $\geq 5 \mathrm{~mm}$.

Exclusion criteria were; patients with immunological diseases such as idiopathic thrombocytopenic purpura, uncontrolled diabetes mellitus, history of chemotherapy or 
radiotherapy, haematological disorder, parafunctional occlusal habit, heavy Smokers and bad oral hygiene.

\section{The Implant system}

DIO IMPLANTS ${ }^{\circledR}$ (DIO, DIO, Korea) They are cylindrical pure titanium with BioTite-H surface which is an electrochemically deposited calcium phosphate coating based upon a biomimetic process in which implants are coated in an electrolytic bath with a $15 \pm 5 \mu \mathrm{m}$ thin bioactive layer of a calcium phosphate composite. BioTite- $\mathrm{H}$ is a composition of the two calcium phosphates Brushite $>95 \%$ and hydroxyapatite $(\mathrm{HA}) \leq 5 \%$. BioTite-H Implant is coated with Resorbable Blast Media (RBM) coating in doublethread area (2mm). RBM surface treatment is a blasting process using absorbable particles that mainly consist of hydroxyapatite, betaTricalcium Phosphate (TCP) and Calcium Pyrophosphate (CPP). It provides the even roughness without leaving embedded debris and acid residue on the implant surface. They are available in different diameters in order to meet the different alveolar ridge widths.Each implant is individually sealed in a gamma-sterilized double safety package and sterility is assured for five years.

\section{The Bone graft}

BoneMedik-DM ${ }^{\circledR}$ (Bone medic, Meta-biomed, Korea) is a bioactive bone graft material, which is resorbable and replaceable by new bone. BoneMedik-DM consists of twophases of ceramics: silicon contained coralline $60 \%$ hydroxyapatite and $40 \%$ beta-tricalcium phosphate. BoneMedik-DM is derived from the exoskeleton of marine coral which has a natural trabecular structure. BoneMedikDM consists of large pores which run parallel to the major growth axis and smaller interconnecting pores which run perpendicular to the major growth axis. This structure is similar with the multidirectional, interconnected porosity of cancellous bone.

\section{Preparation of sticky bone (9)}

20CC of patient's venous blood was taken from patient's forearm vein, and the blood was divided equally to noncoated vacutainers to obtain autologous fibrin glue (AFG), which was used to make sticky bone and to glass coated test tubes without anticoagulants to obtain CGF layer.

The blood in the test tubes was centrifuged at 24002700rpm with a rotor turning at alternated and controlled speed for 12 minutes. The centrifugation time for AFG varied from 2-12 minutes.

The centrifuge was stopped after two minutecentrifugation and AFG tube was taken out of the centrifuge first. The non-coated tube showed two different layers. The upper layer was autologous fibrin glue (AFG) layer and red blood cells were collected in bottom layer which was discarded.

The upper AFG was obtained with syringe and mixed with particulate bone powder (Bone medic, Meta-biomed, Korea) and allowed for five-ten minutes for polymerization in order to produce sticky bone which is yellow colored (Figure 1).

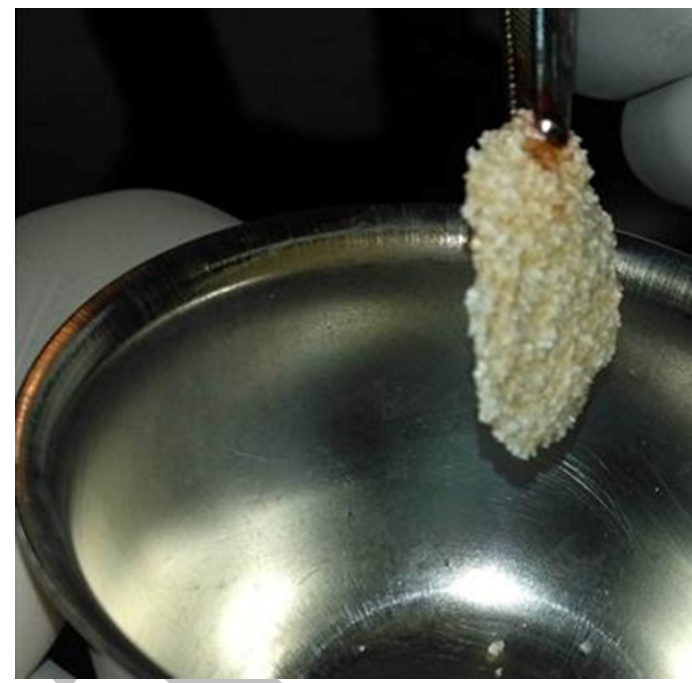

Figure 1: Sticky bone.

Continued centrifugation to prepare CGF. After centrifugation, silica coated tube shows three different layers. The upper most layer was platelet poor plasma, and the middle layer was fibrin buffy coat layer represented by a very large and dense polymerized fibrin block containing the concentrated growth factors. The bottom layer was red blood cell layer.

The concentrated growth factors layer was compressed to be converted to concentrated growth factor enriched fibrin membrane.

\section{Preoperative phase}

Initial treatment planning was started with a thorough clinical examination and cone beam computed tomography (CBCT) to determine alveolar ridge dimensions (figure 2a, 2b).

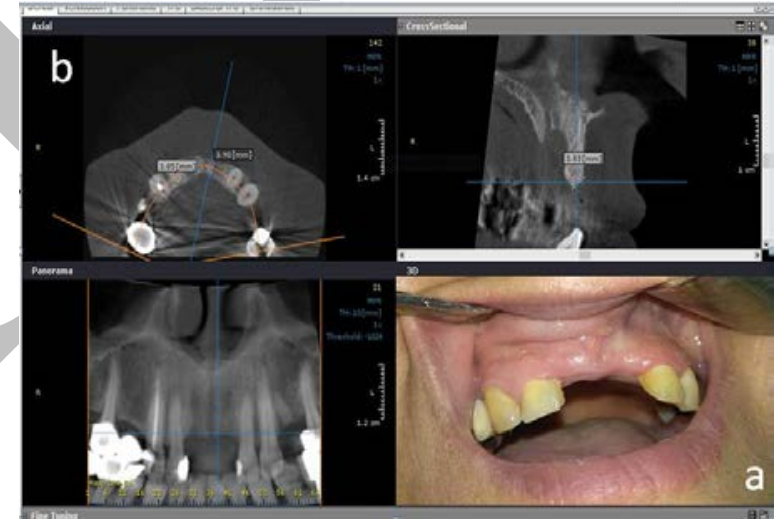

Figure 2: a-Clinical preoperative phase.

b-Preoperative CBCT showing ridge dimensions

\section{Surgical phase}

The patient rinsed thoroughly for 30 seconds with $0.12 \%$ Chlorhexidine mouthwash (Listermix Plus, Sigma, Egypt) 2 minutes prior to surgery. Local anesthesia was achieved by administration of 2\% Mepivacaine $\mathrm{HCl}$ with 1:20,000 Levonordefrin (Mepacaine-L, Alexandria Pharmaceutical Co., Egypt). A fully-reflected mucoperiosteal flap was made and the alveolar ridge was exposed. Preparation of the implant site was done using the specific sequence of drills under copious external cooling. Endosseous implants (Dio, Dio, Korea) was inserted ensuring sufficient primary stability using torque wrench with mean value $25 \mathrm{Ncm}$ (Figure 3a). The prepared sticky bone was grafted over dehiscence defect and covered by concentrated growth 
factors enriched fibrin membrane (Figure 3b). The wound was closed primarily.
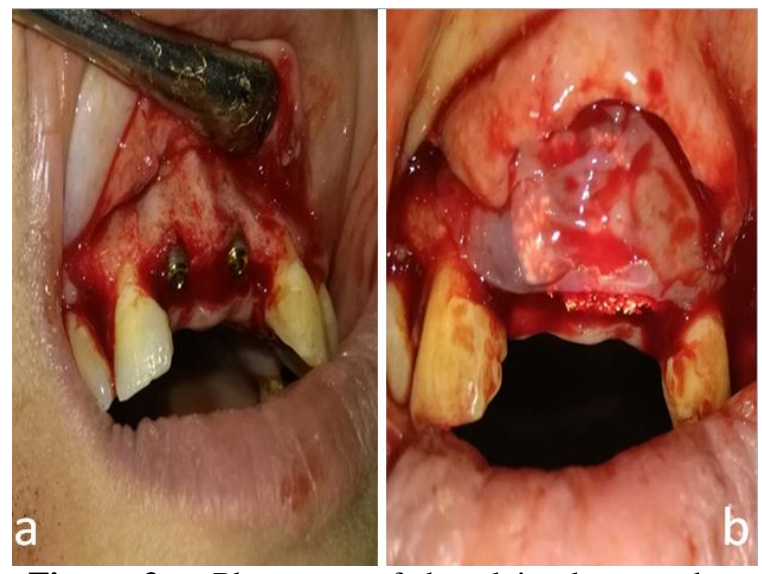

Figure 3: a-Placement of dental implants and resultant labial dehiscence defect.

b- Dehiscence defect augmented with sticky bone and CGF enriched fibrin membrane.

\section{Post-operative phase}

Amoxicillin-Clavulanic acid (Augmentin, GSK, UK) antibiotic (1 gram/12 hours) was prescribed for 7 days. Diclophenac potassium (Cataflam, Novartis, Switzerland) (50 mg/8 hours) was prescribed for 3 days.

Chlorhexidine mouthwash (Listermix Plus, Sigma, Egypt) (every 8 hours) was prescribed to be used starting the day following the surgery. Sutures were removed 7 days after surgery.

\section{Evaluation phase}

\section{Clinical evaluation}

\section{After first-stage surgery}

Overlying mucosa was examined for any signs of inflammation, infection or dehiscence at monthly intervals. Pain: Pain was evaluated on the second day, after 45 days and after six months through visual analogue scale(VAS) from one to ten where pain intensity score scaled from zero (No pain) to ten (unbearable pain) according to Visual Analogue Scale (10).

\section{second-stage surgery After}

Six months post-operatively, the implant was examined for:

Pain: Pain was evaluated after six months

Stability using resonance frequency analysis (osstell ${ }^{\mathrm{TM}}$, osstellAB,sweden)

\section{Implant mobility (10)}

Mobility was tested using back and forth pressure by two instrument handles. Implant mobility indicates lack of osseointegration. Therefore, mobility was used as a specific diagnostic test pointing to loss of osseointegration and being decisive in making the decision to remove the affected implant.

The clinical implant mobility scale is:

Scale 0: Absence of clinical mobility with $500 \mathrm{~g}$ in any direction.

Scale 1: Slight detectable horizontal movement.

Scale 2: Moderate visible horizontal mobility up to 0.5 $\mathrm{mm}$.

Scale 3: Severe horizontal movement greater than $0.5 \mathrm{~mm}$. Scale 4: Visible moderate to severe horizontal movement and any visible vertical movement.

\section{Radiographic evaluation}

A preoperative CBCT was made for the assessment of alveolar ridge dimensions and angulation.

Immediate Postoperative CBCT was made to confirm the position, angulation of the implant fixtures and the size of vertical dehiscence defect measured from the implant platform to the apical end of labial defect (figure 6).

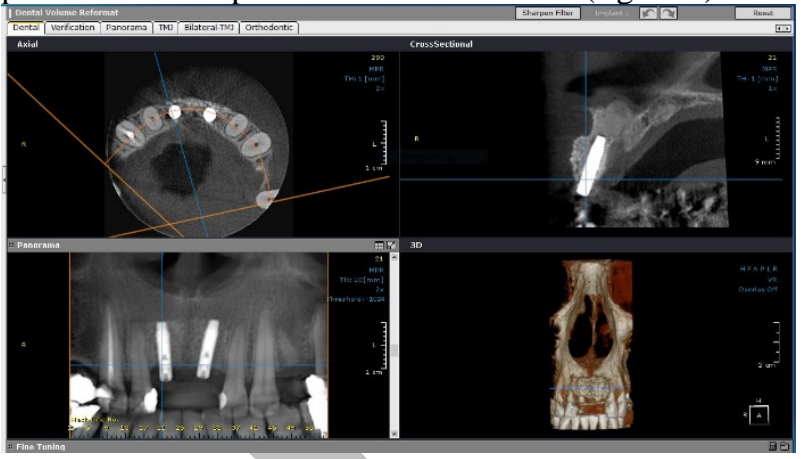

Figure 6: Postoperative CBCT showing sufficiently recovered dehiscence defect.

Six months postoperatively, CBCT was taken and the change in defect dimension was compared to immediate postoperative measures using computer software (OnDemand3D, Cybermed Inc., Korea).

\section{Prosthetic phase}

Final prosthetic treatment (porcelain fused to metal crown) was performed after six months (Figure 7).

\section{Statistical Analysis}

Data were fed to the computer and analyzed using IBM SPSS software package version 20.0. (Armonk, NY: IBM Corp) (2) Qualitative data were described using number and percent. The Kolmogorov-Smirnov test was used to verify the normality of distribution Quantitative data were described using range (minimum and maximum), mean, standard deviation and median. Significance of the obtained results was judged at the $5 \%$ level. Wilcoxon signed ranks test was used For abnormally distributed quantitative variables, to compare between two periods.

\section{RESULTS}

\section{Clinical evaluation}

\section{After first-stage surgery}

Overlying mucosa did not show any signs of inflammation or infection.

After surgery, patient experienced slight to mild pain (one to three) at surgical site for one to three days duration.

After second-stage surgery Pain

On second stage surgery i.e. after six months no patient experienced any sign of pain.

\section{Implant stability}

Data were collected regarding implant stability for all implants (Table 1). The mean of average implant stability was 59.89 ISQ \pm 3.92 with minimum value of 56.0 ISQ and maximum value of 68.0 ISQ.

\section{Implant mobility}

All over the evaluation period, none of implants showed any sign of mobility (i.e. mobility score was 0 )..

\section{Radiographic evaluation}

Preoperative assessment of alveolar ridge dimensions Alveolar ridge width with minimum value of $2.95 \mathrm{~mm}$ and maximum value of $4.04 \mathrm{~mm}$ and mean $3.32 \mathrm{~mm}$. 


\section{Dehiscence defect assessment immediately post-operative}

Vertical dehiscence defect measurements with mean of 5.27 $\mathrm{mm} \pm 0.47 \mathrm{SD}$ with maximum value of $6.0 \mathrm{~mm}$ and minimum value of $4.50 \mathrm{~mm}$.

Dehiscence defect assessment 6 months post-operative

Vertical dehiscence defect was sufficiently recovered in 5 implant-sites while in the other 6 sites it was decreased with mean of $1.25 \mathrm{~mm} \pm 0.69 \mathrm{SD}$ with maximum value of 2.0 $\mathrm{mm}$ and minimum value of $0.5 \mathrm{~mm}$ (Figure 8), i.e the defect coverage in 6 implants occurred with mean of $4.59 \mathrm{~mm} \pm$ $0.49 \mathrm{SD}$ with minimum value of $4.0 \mathrm{~mm}$ and maximum value of $5.0 \mathrm{~mm}$ (Table 2).

Table (1): Distribution of the studied cases according to ostell readings $(n=11)$.

\begin{tabular}{||l|c|c|c||}
\hline \multicolumn{1}{|c|}{ Ostell readings } & Min. - Max. & Mean \pm SD. & Median \\
\hline Labial & $55.0-69.0$ & $59.27 \pm 4.29$ & 58.0 \\
\hline Palatal & $55.0-69.0$ & $59.18 \pm 4.12$ & 58.0 \\
\hline Mesial & $55.0-69.0$ & $60.82 \pm 4.40$ & 60.0 \\
\hline Distal & $55.0-65.0$ & $60.27 \pm 3.74$ & 58.0 \\
\hline Average & $56.0-68.0$ & $59.89 \pm 3.92$ & 58.50 \\
\hline
\end{tabular}

Table (2): Distribution of the studied cases according to different parameters.

\begin{tabular}{|l|c|c|c|c||}
\hline & $\mathbf{N}$ & $\begin{array}{c}\text { Min. - } \\
\text { Max. }\end{array}$ & Mean \pm SD. & Median \\
\hline $\begin{array}{l}\text { Resultant defect } \\
\text { immediately post-operative } \\
\text { (mm.) }\end{array}$ & $\mathbf{1 1}$ & $4.50-6.0$ & $5.27 \pm 0.47$ & 5.0 \\
\hline $\begin{array}{l}\text { Area covered from defect 6 } \\
\text { months post-operative } \\
\text { (mm.) }\end{array}$ & $\mathbf{1 1}$ & $4.0-5.0$ & $4.59 \pm 0.49$ & 5.0 \\
$\begin{array}{l}\text { Remaining defect area 6 } \\
\text { months post-operative } \\
\text { (mm.) }\end{array}$ & $\mathbf{6}$ & $0.50-2.0$ & $1.25 \pm 0.69$ & 1.5 \\
\hline
\end{tabular}

Assessment of bone density for ridge area covered with (Sticky Bone)

Data were collected regarding bone density for Concentrated Growth Factors (CGF) Enriched Bone Graft Matrix (Sticky Bone) mean values and standard deviation at immediate post-operative and six months post-operative (Table 3).

Table (3):Comparison between Immediately and 6 months post-operative according to bone density $(\mathrm{n}=11)$.

\begin{tabular}{||c|c|c|c|c||}
\hline \multirow{2}{*}{ Bone density } & \multicolumn{2}{|c|}{ Post-operative } & \multirow{2}{*}{$\mathbf{Z}$} & P \\
\cline { 2 - 5 } & Immediately & $\mathbf{6}$ months & & \\
\hline $\begin{array}{l}\text { Min. }- \\
\text { Max. } \\
\text { Mean } \pm \text { SD. } \\
\text { Median }\end{array}$ & $415.0-746.0$ & $1371.0-1816.0$ & & \\
\hline $\begin{array}{l}\text { \% of } \\
\text { Change }\end{array}$ & 468.0 & 1548.9 & $34^{*}$ & $0.003^{*}$ \\
\hline
\end{tabular}

At immediate post-operatively, the mean bone density for Concentrated Growth Factors (CGF) Enriched Bone Graft Matrix (Sticky Bone) value was 531.45 HU \pm 116.3 with a minimum recorded value $415.0 \mathrm{HU}$ and a maximum recorded value of 746.0. HU.

On the sixth month, the mean bone density for Concentrated Growth Factors (CGF) Enriched Bone Graft Matrix (Sticky Bone)was 1558.9 HU \pm 154.2 with a minimum recorded value of $1371.0 \mathrm{HU}$ and a maximum recorded value of 1816.0. HU.

\section{DISCUSSION}

The purpose of this study was to evaluate a new technique for dealing with narrow ridge by placement of dental implant and covering the resultant labial dehiscence defect with a special concentrated growth factors (CGF) enriched bone graft concentrated growth factors (CGF) is an immune and platelet concentrate collecting on a single fibrin membrane containing all constituents of a blood sample which are favourable for healing and immunity (11).

CGF, like PRF, does not require the addition of bovine thrombin or any anticoagulants. Furthermore, altered protocols in obtaining the blood sample and in the centrifuging procedure compare with PRF. Unlike PRF however, CGF uses variable rpm from 2400-2700 rpm to separate cells in the venous blood, resulting in fibrin rich blocks that are much larger, denser and richer in growth factors than common PRF (12).

This study was conducted on 11 implants with anterior alveolar ridge defect. Patients were selected from the outpatient clinic of Oral and Maxillofacial Surgery Department, Faculty of Dentistry, Alexandria University.

At first stage surgery the patients experienced slight pain at the surgical site which disappeared completely after the 2nd and 3rd postoperative days, while patients didn't experience any sign of pain in the second stage. These limited postoperative signs after using the second generation of platelets concentrates are in agreement with studies done in 2006 by Choukroun et al. (13), in 2007 by Grossi et al. (14) and in 2008 by Baqain et al (15). Although there are obvious differences between the extraction of a tooth and the placement of a dental implant, the mucosal and alveolar tissues are traumatised in both procedures, resulting in similar mechanisms of inflammatory pain.

Regarding implant mobility, no clinical mobility was detected in any of the implants throughout the follow up period. This was confirmed by radiographic evaluation that revealed absence of peri-implant radiolucency. This indicates proper osseointegration of all implants. The absence of implant mobility is considered to be important criteria for implant success as mentioned in 2005 by Porter and Von Fraunhofer (16).

In our study implant stability was assessed by osstell device. The mean of implant stability quotient (ISQ) was $59.89 \pm 3.92$ ISQ. This was in agreement with Shiigai in 2007 (17) and Anitha et al in 2014 (18) who mentioned that the primary stability of implant with ISQ more than 62 considered to be suitable.

In the present study there was marked decrease in bone dehiscence defect after six months follow up, which is in a line with Several studies that evaluate the effect of PRF with different grafting material. Choukroun et al. (19) reported that the combination of PRF which contain CGF with bone 
graft (allograft) has the ability to induce more bone regeneration.

Regarding mean values of bone density for grafting material, there was marked increase in bone density after six month follow up .This is in agreement with Ozemir et al (20) who demonstrated that the use of PRF can increase the quality (density) of the newly formed bone and enhance the rate of new bone formation and this may be explained by the presence of concentrated growth factor in the PRF which is in agreement with our result.

In accordance with our study Lobo et al (21) reported the presence of porosity and bioactive surface in the biphasic calcium phosphate when used in combination with PRF facilitating cell attachment, proliferation and differentiation which in turn provides more biocompatible, osteoconductive and osteoinductive combination leading to more new bone formation than when BCP used alone. This is due to the formation of stable scaffold allowing for vascular in growth inside the scaffold.

Kumar and Shubhashini (22) reported that the second generation platelet concentrate called platelet-rich fibrin (PRF) has been widely used to accelerate soft and hard tissue healing this may be due to the presence of inflammatory cytokines, healing cytokines and growth factors. This result is in accordance with our results.

Roy et al., (23) demonstrated that the use of PRF matrix effectively induce endothelial cell proliferation and improved wound angiogenesis in chronic wound. This is in line with our results.

In the current study CGF membrane was utilized to cover the CGF enriched bone graft. It shows better regenerative capacity and higher versatility. The resulting fibrin clot/block is of a higher quality due to the concentration of fibrinogen, factor XIII and thrombin that is obtained. Factor XIIIa, which is activated by thrombin, cross links the fibrin clot to increase stability, strength and protection against plasmin mediated degradation. Clinically, this translates to a clot with higher tensile strength (1.5 kg after 1 hour vs $500 \mathrm{gm})$, adhesive strength, and decrease in haemostatic time (105 secs vs 360 secs)(24). CGF enriched bone matrix with CGF enriched fibrin membrane could be used as an alternative to titanium mesh or block bone procedure. They are moldable, so well adapted over various shapes of bony defect. Micro and macro movement of grafted bone is prevented, so the volume of bone augmentation is maintained during healing period. Fibrin network entraps platelets and leukocytes to release growth factors, so bone regeneration and soft tissue regeneration is accelerated (25).

\section{CONCLUSION}

The combination of CGF enriched bone graft matrix (sticky bone) and CGF enriched fibrin membrane can be very effective for reconstruction of alveolar bone defect.

Sticky bone not only enhance the rate of new bone formation, but also increase the quality (density) of the newly-formed bone.

\section{REFERENCES}

1. Widmann G, Bale RJ. Accuracy in computer-aided implant surgery--a review. Int $\mathrm{J}$ Oral Maxillofac Implants. 2006;21:305-13.

2. Laurencin C, Khan Y, El-Amin SF. Bone graft substitutes. Expert Rev Med Devices. 2006;3:49-57.
3. Simion M, Jovanovic SA, Trisi P, Scarano A, Piattelli A. Vertical ridge augmentation around dental implants using a membrane technique and autogenous bone or allografts in humans. Int J Periodontics Restorative Dent. 1998;18:8-23.

4. Rosenkranz S, Kazlauskas A. Evidence for distinct signaling properties and biological responses induced by the PDGF receptor alpha and beta subtypes. Growth Factors. 1999;16:201-16.

5. Tozum TF, Demiralp B. Platelet-rich plasma: a promising innovation in dentistry. J Can Dent Assoc. 2003;69:664.

6. Marx RE, Carlson ER, Eichstaedt RM, Schimmele SR, Strauss JE, Georgeff KR. Platelet-rich plasma: Growth factor enhancement for bone grafts. Oral Surg Oral Med Oral Pathol Oral Radiol Endod. 1998;85:638-46.

7. Plachokova AS, Nikolidakis D, Mulder J, Jansen JA, Creugers NH. Effect of platelet-rich plasma on bone regeneration in dentistry: a systematic review. Clin Oral Implants Res. 2008;19:539-45.

8. Plachokova AS, Nikolidakis D, Mulder J, Jansen JA, Creugers NH. Effect of platelet-rich plasma on bone regeneration in dentistry: a systematic review. Clin Oral Implants Res. 2008;19:539-45.

9. Intini G. The use of platelet-rich plasma in bone reconstruction therapy. Biomaterials. 2009;30:4956-66.

10. Bijur PE, Silver W, Gallagher EJ. Reliability of the visual analog scale for measurement of acute pain. Acad Emerg Med. 2001;8:1153-7.

11. Mosesson MW, Siebenlist KR, Meh DA. The structure and biological features of fibrinogen and fibrin. Ann NY Acad Sci. 2001;93:11-30.

12. Sohn DS, Moon JW, Moon YS, Park JS, Jung HS. The use of concentrated growth factors (CGF) for sinus augmentation. Implant J. 2009;38:25-35.

13. Choukroun J, Diss A, Simonpieri A, Girard MO, Schoeffler C, Dohan SL, et al. Platelet-rich fibrin (PRF): A secondgeneration platelet concentrate.Part IV: Clinical effects on tissue healing. Oral Surg Oral Med Oral Pathol Oral Radiol Endod. 2006;101:E56-60.

14. Grossi GB, Maiorana C, Garramone RA, Borgonovo A, Creminelli L, Santoro F. Assesing postoperative discomfort after third molar surgery:A prospective study. J Oral Maxillofacial Surg. 2007;65:901-17.

15. Baqain Z, Karaky AA, Sawair F, Khraisat A, Duaibis R, Rajab LD. Frequency estimates and risk factors for post operative morbidity after third molar removal:A prospective cohort study. J Oral Maxillofac Surg. 2008;66(11):2276-83.

16. Porter JA, von Fraunhofer JA. Success or failure of dental implants? A literature review with treatment considerations. General Dent. 2005;53:423-32.

17. Shiigai T. Pilot study in the identification of stability values for determining immediate and early loading of implants. J Oral Implantol. 2007;33:13-22.

18. Anitha K, Kumar SS, Babu MRR, Candamourty R, Thirumurugan. Immediate implants in anterior maxillary arch. J Nat Sci Biol Med. 2014;5:82-9.

19. Choukroun J, Diss A, Simonpieri A, Girard MO, Schoeffler C, Dohan SL, et al. Platelet-rich fibrin (PRF): a secondgeneration platelet concentrate. Part V: histologic evaluations of PRF effects on bone allograft maturation in sinus lift. Oral Surg Oral Med Oral Pathol Oral Radiol Endod. 2006;101:299-303. 
20. Ozdemir H, Ezirganli S, Isa Kara M, Mihmanli A, Baris E. Effects of platelet rich fibrin alone used with rigid titanium barriers. Arch Oral Biol. 2013;58:537-44.

21. Lobo SE, Wykrota FH, Oliveira AC, Kerkis I, Mahecha GB, Alves HJ. Quantification of bone mass gain in response to the application of biphasic bioceramics and platelet concentrate in critical-size bone defects. J Mater Sci Mater Med. 2009;20:1137-47.

22. Kumar RV, Shubhashini N. Platelet rich fibrin: A new paradigm in periodontal regeneration. Cell Tissue Bank. 2013;14:453-63.

23. Roy S, Driggs J, Ehgharably H, Biswas S, Findley M, Khanna S, et al. Platelet rich fibrin matrix improves wound angiogenesis via inducing endothelial cell proliferation. Wound Repair Regen. 2011;19:753-66.

24. Mansour P, Kim P. Use of concentrated growth factors (CGF) in implantology. Silfradent. 2015 (Apr).

25. Kim J. Utilization of autologous concentrated growth factors (CGF) enriched bone graft matrix (sticky bone) and CGF-Enriched fibrin membrane in implant dentistry. 2015 (Dec); 7(10): 11-28.

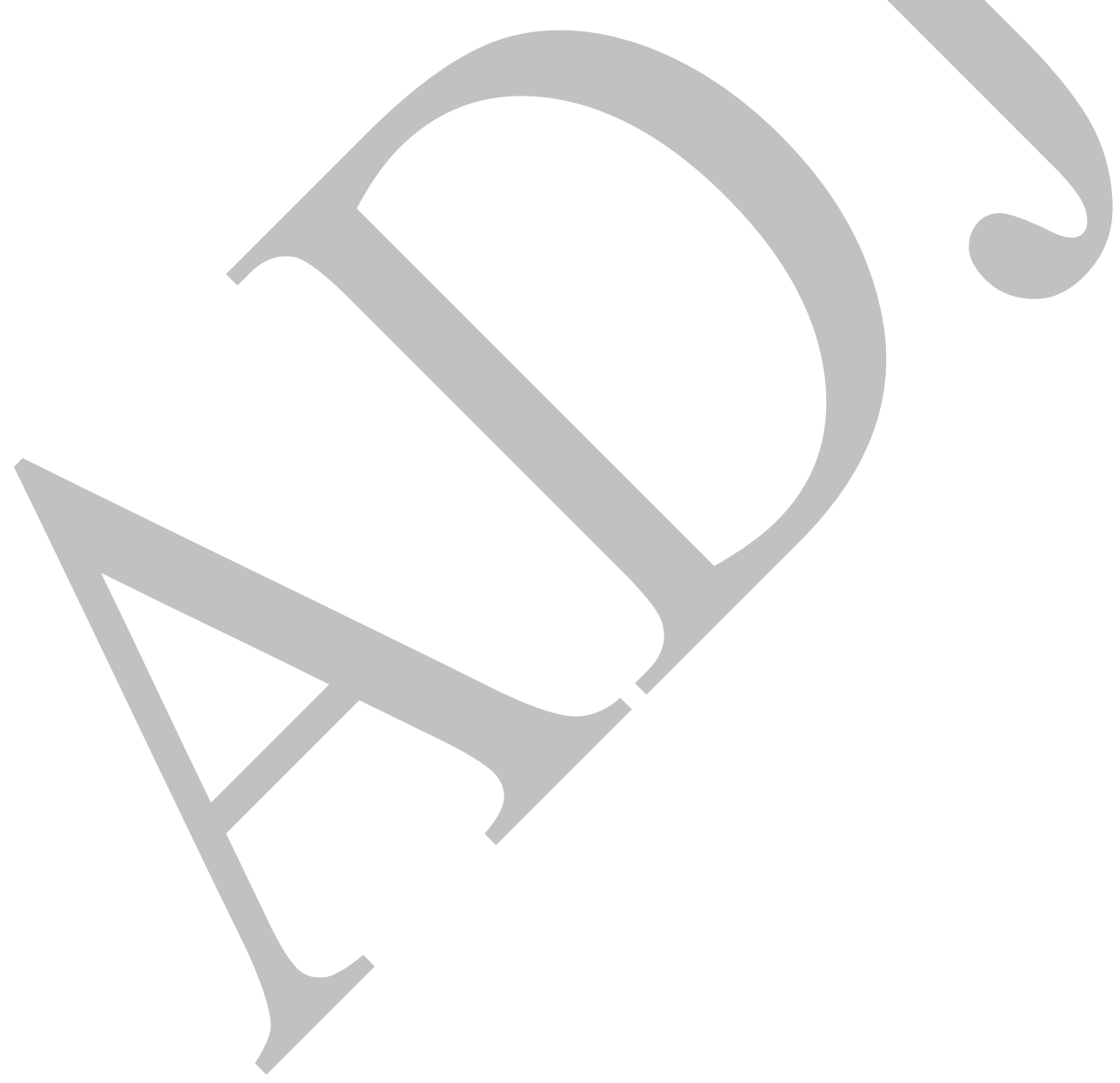

